\title{
De novo pulmonary small cell carcinomas and large cell neuroendocrine carcinomas harboring EGFR mutations: Lack of response to EGFR inhibitors
}

\section{Citation}

Le, Xiuning, Neelam V. Desai, Adnan Majid, Rebecca S. Karp, Mark S. Huberman, Deepa Rangachari, Michael S. Kent, et al. 2015. “De Novo Pulmonary Small Cell Carcinomas and Large Cell Neuroendocrine Carcinomas Harboring EGFR Mutations: Lack of Response to EGFR Inhibitors." Lung Cancer 88 (1) (April): 70-73. doi:10.1016/j.lungcan.2015.02.003.

\section{Published Version}

10.1016/j.lungcan.2015.02.003

\section{Permanent link}

http://nrs.harvard.edu/urn-3:HUL.InstRepos:37047104

\section{Terms of Use}

This article was downloaded from Harvard University's DASH repository, and is made available under the terms and conditions applicable to Open Access Policy Articles, as set forth at http:// nrs.harvard.edu/urn-3:HUL.InstRepos:dash.current.terms-of-use\#OAP

\section{Share Your Story}

The Harvard community has made this article openly available.

Please share how this access benefits you. Submit a story.

\section{Accessibility}




\section{Abstract}

Introduction-Epidermal growth factor receptor (EGFR) mutations are present in 10-20\% of all non-small-cell lung cancers and predict for response to EGFR tyrosine kinase inhibitors (TKIs). However, the incidence of these mutations and their ability to predict response to TKIs in highgrade pulmonary neuroendocrine carcinomas [i.e. small cell lung cancer (SCLC) and large cell neuroendocrine carcinoma (LCNEC)] is unknown.

Methods-The presence of EGFR mutations, clinicopathologic and anti-cancer therapy response data were retrospectively compiled and analyzed from a cohort of 608 patients-lung tumors to identify EGFR mutated high-grade pulmonary neuroendocrine carcinomas. We identified 126 $E G F R$-mutated (21.8\% of 578 successful genotyped cases) lung cancers and only 2 (1.6\%) were high-grade neuroendocrine carcinomas.

\footnotetext{
(c) 2015 Published by Elsevier Ltd.

"Correspondence to: Daniel B. Costa, MD, PhD - Division of Hematology/Oncology, Beth Israel Deaconess Medical Center, 330 Brookline Av., Boston, MA 02215 Phone: 617-667-9236, Fax: 617-975-5665, dbcosta@ bidmc.harvard.edu Or Paul A. VanderLaan, MD, PhD - Department of Pathology, Beth Israel Deaconess Medical Center, 330 Brookline Av., Boston, MA 02215 Phone: 617-667-5762, Fax: 617-667-7120, pvanderl@bidmc.harvard.edu.

Publisher's Disclaimer: This is a PDF file of an unedited manuscript that has been accepted for publication. As a service to our customers we are providing this early version of the manuscript. The manuscript will undergo copyediting, typesetting, and review of the resulting proof before it is published in its final citable form. Please note that during the production process errors may be discovered which could affect the content, and all legal disclaimers that apply to the journal pertain.

CONFLICT OF INTEREST STATEMENT

Daniel B. Costa has received consulting fees and honoraria from Pfizer (unrelated to the current work).

Neelam V. Desai, Adnan Majid, Rebecca S. Karp, Mark S. Huberman, Deepa Rangachari, Michael S. Kent, Sidharta P. Gangadharan, Erik Folch, MD and Paul A. VanderLaan have no conflicts to disclose.

No other conflict of interest is stated.
} 
Results-Case one was of a 63 year-old white never smoker woman with extensive stage SCLC harboring EGFR-delL747_P753insS but without EGFR protein expression. After progression on carboplatin/etoposide, the patient was treated with erlotinib and developed progressive disease with a survival $<3$ months from start of erlotinib. Case two was of a 73 year-old Asian 30 packyear smoker man with metastatic LCNEC harboring EGFR-delL747_P753insQS and also lacking EGFR protein expression. The patient received first line therapy with erlotinib and had progressive disease with a survival of 4 months.

Conclusions-The lack of response to EGFR TKIs in EGFR mutated de novo SCLC and LCNEC reported here may indicate that tumor differentiation affects tumor dependency on EGFR as a driver oncogene.

\section{Keywords}

mutation; lung cancer; small cell lung cancer; large cell neuroendocrine carcinoma; EGFR; neversmoker; erlotinib; progression; resistance

\section{INTRODUCTION}

Lung cancer is the leading cause of all cancer deaths worldwide (1). The most frequently encountered primary lung cancers include adenocarcinoma, squamous cell carcinoma, and neuroendocrine carcinomas, with small cell lung cancer (SCLC) and large cell neuroendocrine carcinoma (LCNEC) typifying high-grade neuroendocrine carcinomas. In the last decade, one of the most dramatic advances in the diagnosis and treatment of patients with lung cancer has been the identification of activating mutations of the epidermal growth factor receptor (EGFR) gene in approximately 10-20\% of lung adenocarcinomas (2). These mutations render growth advantage to the cancer cells and their inhibition correlates with responsiveness to EGFR tyrosine kinase inhibitors (TKIs), such as the clinically-available TKIs gefitinib, erlotinib and afatinib (2).

Routine testing for EGFR mutations is now part of evidence-based care for advanced NSCLCs of adenocarcinoma histology (3) but not recommended for squamous cell carcinomas or neuroendocrine lung tumors. EGFR mutations have been previously identified in SCLCs and LCNEC; but the majority of these cases have been described as developing as a rare (approximately $5 \%$ of cases) mechanism of resistance to EGFR TKI therapy for EGFR mutated lung adenocarcinomas (4-7). De novo TKI-naïve SCLCs and LCNECs with classic EGFR mutations are rarely described and their clinical response to EGFR TKIs is largely unknown (8-10). Here, we report the lack of response to erlotinib in de novo SCLC and LCNEC, which implies that tumor differentiation affects tumor dependency on EGFR expression and signaling.

\section{MATERIALS AND METHODS}

\section{Cohort selection}

Patients seen at Beth Israel Deaconess Medical Center (BIDMC) with a diagnosis of lung cancer and whose tumors were genotyped for at least EGFR mutations were identified 
through an ongoing Institutional Review Board approved protocol (11-12); with a data cut off of August $28^{\text {th }} 2014$.

\section{Tumor diagnosis and genotyping}

Following the pathologic diagnosis (including ancillary immunohistochemical staining), the residual tumor material in the formalin-fixed paraffin-embedded (FFPE) tissue blocks were submitted for molecular analysis. EGFR mutation analysis (exons 18 to 21) was performed using standard sequencing (11).

\section{Immunohistochemical (IHC) evaluation of tumors}

Immunohistochemistry for EGFR was performed using the EGFR-D38B1 antibody (Cell Signaling) according to the manufacturer's protocol.

\section{Data collection and medical chart extraction}

Clinical, pathologic, radiographic and tumor genotyping data were collected from chart extraction. Study data were collected and managed using REDCap electronic data capture tools hosted at BIDMC. The complete cohort comprised 608 patients, with 361 women (59.4\%), 158 never smokers (26.0\%), 317 former smokers (52.1\%) and 133 current smokers (21.9\%). 431 patients (71.0\%) had stage IV lung cancer, 527 tumors $(86.7 \%)$ were adenocarcinoma, 51 NSCLC-not otherwise specified (NSCLC-NOS), 19 (3.1\%) squamous cell carcinomas, $3(0.5 \%)$ LCNECs, $3(0.5 \%)$ SCLCs and 5 had a different histology.

\section{RESULTS}

\section{Frequency of EGFR mutations}

Among the 608 cases, 578 tumor samples were successfully genotyped for EGFR mutations. 126 (21.8\% of the 578 cases) tumors harbored EGFR mutations: 122 (96.8\%) tumors were classified as adenocarcinoma, $2(1.6 \%)$ as NSCLC-NOS and $2(1.6 \%)$ as high-grade neuroendocrine tumors (1 SCLC and 1 LCNEC). Only 6 high-grade neuroendocrine carcinomas (3 SCLC and 3 LCNEC) were genotyped at our service. One out of the 3 (1 patient was a never smoker while the other 2 had $<25$ pack-year history of smoking) genotyped SCLCs had an EGFR-delL747_P753insS mutation. One out of 3 (1 patient was a never smoker while the other 2 had $<40$ pack-year history of smoking) genotyped LCNECs harbored an EGFR-delL747_P753insQS mutation.

\section{Case reports and response to EGFR TKI therapy}

Case 1-The patient was a 63-year-old Caucasian female never smoker, who presented with persistent cough and dyspnea. Positron emission tomography-computed tomography (PET-CT) disclosed a right upper lobe and right hilar mass encasing the pulmonary artery, with extensive mediastinal/abdominal lymphadenopathy, liver and bone metastases. Transbronchial biopsy of the right upper lobe mass as well as endobronchial ultrasound guided transbronchial needle aspiration of lymph node stations 4R, 4L, and 7 were positive for small cell carcinoma (Figure 1A-C). The biopsies were devoid of any evidence of a nonsmall-cell component. Genotype disclosed an EGFR-delL747_P753insS mutation. As 
standard therapy for extensive stage small cell carcinoma, the patient received 6 cycles of carboplatin and etoposide chemotherapy with an excellent clinical response and partial radiographic regression. However, 1 month after completion of chemotherapy the patient displayed symptomatic disease progression with central airway compression. A bronchoscopy was performed to stent the airway and biopsies again confirmed small cell histology. Repeat genotyping of the tumor at this time reconfirmed EGFRdelL747_P753insS. Therefore, off label erlotinib $150 \mathrm{mg} /$ day was started. Clinical symptoms did not improve and imaging studies performed within 3 weeks of therapy disclosed progressive disease. IHC staining for EGFR was performed, and demonstrated the virtual absence of staining in tumor cells (Figure 1D). The patient declined second line cytotoxic chemotherapy for small cell lung cancer and died 2 months after initiation of erlotinib.

Case 2-The second patient was a 73-year-old Asian man with a prior 30 pack-year smoking history who presented with dyspnea on exertion and weight loss. A PET-CT showed a spiculated right lower lung mass with multiple enlarged central lymph nodes and an abdominal nodule. MRI of the brain showed four small metastases. Core needle biopsy of the abdominal metastasis demonstrated a poorly differentiated non-small cell carcinoma lacking glandular or squamous differentiation but demonstrating neuroendocrine features, consistent with a pulmonary LCNEC (Figure 2). Genotype disclosed EGFRdelL747_P753insQS. IHC for EGFR disclosed lack of staining in tumor cells (13). He started erlotinib $150 \mathrm{mg} /$ day and this was dose reduced to $100 \mathrm{mg} /$ day due to intolerable rash and diarrhea. The first imaging study was performed 3 months after initiation of erlotinib and disclosed progressive disease with new metastatic sites. Numerous new brain metastases also developed. The patient declined chemotherapy with carboplatin and etoposide, and died 4 months after initiation of erlotinib from progressive tumor burden.

\section{DISCUSSION}

Current evidence-based guidelines for the management of advanced lung cancers recommend analysis of EGFR mutations for all lung adenocarcinomas but discourage testing for other tumor histologies $(3,12)$. Few, if any, cases of neuroendocrine lung tumors are sent for $E G F R$ genotype in routine clinical practice. In our institution, <1\% of all cases genotyped were high-grade neuroendocrine carcinomas; and invariably the decision to send these tumors for EGFR mutation analysis hinged in the perceived lack of noteworthy smoking history of patients (i.e., the smoking history was discordant with the typical pattern of significant smoking seen in cases of small cell lung cancer). Our knowledge on the frequency of classic EGFR mutations in de novo high-grade neuroendocrine carcinomas of lung origin in never or light smokers originates from limited cohorts of patients-tumors (8, 9). We identified classic EGFR mutations in 2 out of 6 (33.3\%) high-grade neuroendocrine carcinomas. It is possible that the frequency of EGFR mutations and other known driver mutations in de novo SCLCs and LCNECs (either pure or of mixed histology) from never or light smokers is in fact not low (9), but under recognized due to current testing guidelines that discourage routine day-to-day genotype of these tumors. 
The clinical implications and the predictive power of EGFR mutations in de novo highgrade neuroendocrine tumors of the lung also remain underreported. In our two cases ( 1 of SCLC and 1 of LCNEC), neither patient had a response to the EGFR TKI erlotinib at its usual doses. We are aware of 2 other cases of de novo EGFR mutated SCLCs that did not respond to the EGFR TKI erlotinib (9), and we were unable to identify in the literature a case of pure SCLC with a confirmed response to EGFR TKIs. One report of a LCNEC harboring an exon 19 deletion EGFR mutation (delL747_A755insAT) and a response to gefitinib $250 \mathrm{mg} / \mathrm{day}$ was reported, however the tumor was diagnosed in a small skin biopsy sample, the patient was a never smoker and the tumor mitotic rate was low; raising the possibility that this could have been a mixed tumor or NSCLC-NOS (14). Additional reports are necessary to determine if most $E G F R$ mutated high-grade neuroendocrine carcinomas (pure or mixed with other NSCLCs histologies) are intrinsically insensitive to EGFR TKIs.

The mechanism of resistance to TKIs in de novo SCLCs and LCNECs is intriguing. We have learned that in a subset of patients with EGFR mutated lung adenocarcinoma responsive to EGFR TKIs, small cell carcinoma can subsequently be found at time of acquired resistance to TKI therapy (4-7). The underlying biological basis for this proposed phenomenon have recently been elucidated by the Thoracic Oncology group at Massachusetts General Hospital, and involves the transition to a neuroendocrine/small cell lung cancer genomic background of the EGFR mutated cancer with subsequent silencing of EGFR expression (13). Intriguingly, the EGFR mutated SCLC and NSCLC cases reported here did not significantly express EGFR (protein) despite the presence of the EGFR mutation in the genomic material. Since EGFR mutated NSCLCs abundantly express EGFR mutant proteins $(4,15)$ the lack of EGFR expression in our cases suggests that the translation of $E G F R$ from DNA/RNA to protein may be hampered in tumors with de novo high-grade neuroendocrine phenotypes.

In summary, we describe cases of SCLC and LCNEC harboring EGFR mutations without clinical/radiographic response to an EGFR TKI.

\section{ACKNOWLEDMENTS}

This work was funded in part through an American Cancer Society grant RSG 11-186 (DBC) and a National Cancer Institute grant CA090578 (DBC).

We would like to thank all current and former members of the Thoracic Oncology Clinic at Beth Israel Deaconess Medical Center, and our patients.

\section{REFERENCES}

1. Siegel R, Ma J, Zou Z, et al. Cancer statistics, 2014. CA Cancer J Clin. 2014; 64:9-29. [PubMed: 24399786]

2. Gerber DE, Gandhi L, Costa DB. Management and future directions in non-small cell lung cancer with known activating mutations. Am Soc Clin Oncol Educ Book. 2014:e353-e365. [PubMed: 24857124]

3. Lindeman NI, Cagle PT, Beasley MB, et al. Molecular Testing Guideline for Selection of Lung Cancer Patients for EGFR and ALK Tyrosine Kinase Inhibitors: Guideline from the College of American Pathologists, International Association for the Study of Lung Cancer, and Association for Molecular Pathology. J Thorac Oncol. 2013; 8:823-859. [PubMed: 23552377] 
4. Sequist LV, Waltman BA, Dias-Santagata D, et al. Genotypic and histological evolution of lung cancers acquiring resistance to EGFR inhibitors. Sci Transl Med. 2011; 3:75ra26.

5. Zakowski MF, Ladanyi M, Kris MG. EGFR mutations in small-cell lung cancers in patients who have never smoked. N Engl J Med. 2006; 355:213-215. [PubMed: 16837691]

6. Alam N, Gustafson KS, Ladanyi M, et al. Small-cell carcinoma with an epidermal growth factor receptor mutation in a never-smoker with gefitinib-responsive adenocarcinoma of the lung. Clin Lung Cancer. 2010; 11:E1-E4. [PubMed: 20837450]

7. Norkowski E, Ghigna MR, Lacroix L, et al. Small-cell carcinoma in the setting of pulmonary adenocarcinoma: new insights in the era of molecular pathology. J Thorac Oncol. 2013; 8:12651271. [PubMed: 24457237]

8. Kurahara Y, Kawaguchi T, Tachibana K, et al. Small-cell lung cancer in never-smokers: a case series with information on family history of cancer and environmental tobacco smoke. Clin Lung Cancer. 2012; 13:75-79. [PubMed: 21729654]

9. Varghese AM, Zakowski MF, Yu HA, et al. Small-cell lung cancers in patients who never smoked cigarettes. J Thorac Oncol. 2014; 9:892-896. [PubMed: 24828667]

10. Rekhtman N, Tafe LJ, Chaft JE, et al. Distinct profile of driver mutations and clinical features in immunomarker-defined subsets of pulmonary large-cell carcinoma. Mod Pathol. 2013; 26:511522. [PubMed: 23196793]

11. VanderLaan PA, Yamaguchi N, Folch E, et al. Success and failure rates of tumor genotyping techniques in routine pathological samples with non-small-cell lung cancer. Lung Cancer. 2014; 84:39-44. [PubMed: 24513263]

12. Yamaguchi N, VanderLaan PA, Folch E, et al. Smoking status and self-reported race affect the frequency of clinically relevant oncogenic alterations in non-small-cell lung cancers at a United States-based academic medical practice. Lung Cancer. 2013; 82:31-37. [PubMed: 23932486]

13. Niederst MJ, Sequist LV, Poirier JT, et al. RB loss in resistant EGFR mutant lung adenocarcinomas that transform to small cell lung cancer. Nat Commun. 2014 in press.

14. De Pas TM, Giovannini M, Manzotti M, et al. Large-cell neuroendocrine carcinoma of the lung harboring EGFR mutation and responding to gefitinib. J Clin Oncol. 2011; 29:e819-e822. [PubMed: 22042963]

15. Costa DB, Halmos B, Kumar A, et al. BIM mediates EGFR tyrosine kinase inhibitor-induced apoptosis in lung cancers with oncogenic EGFR mutations. PLoS Med. 2007; 4:1669-1679. [PubMed: 17973572] 


\section{Highlights}

- EGFR mutations occur in some high-grade neuroendocrine lung cancers

- EGFR protein is not expressed in EGFR mutated neuroendocrine lung cancers

- Patients with neuroendocrine lung cancers don't respond to EGFR inhibitors 

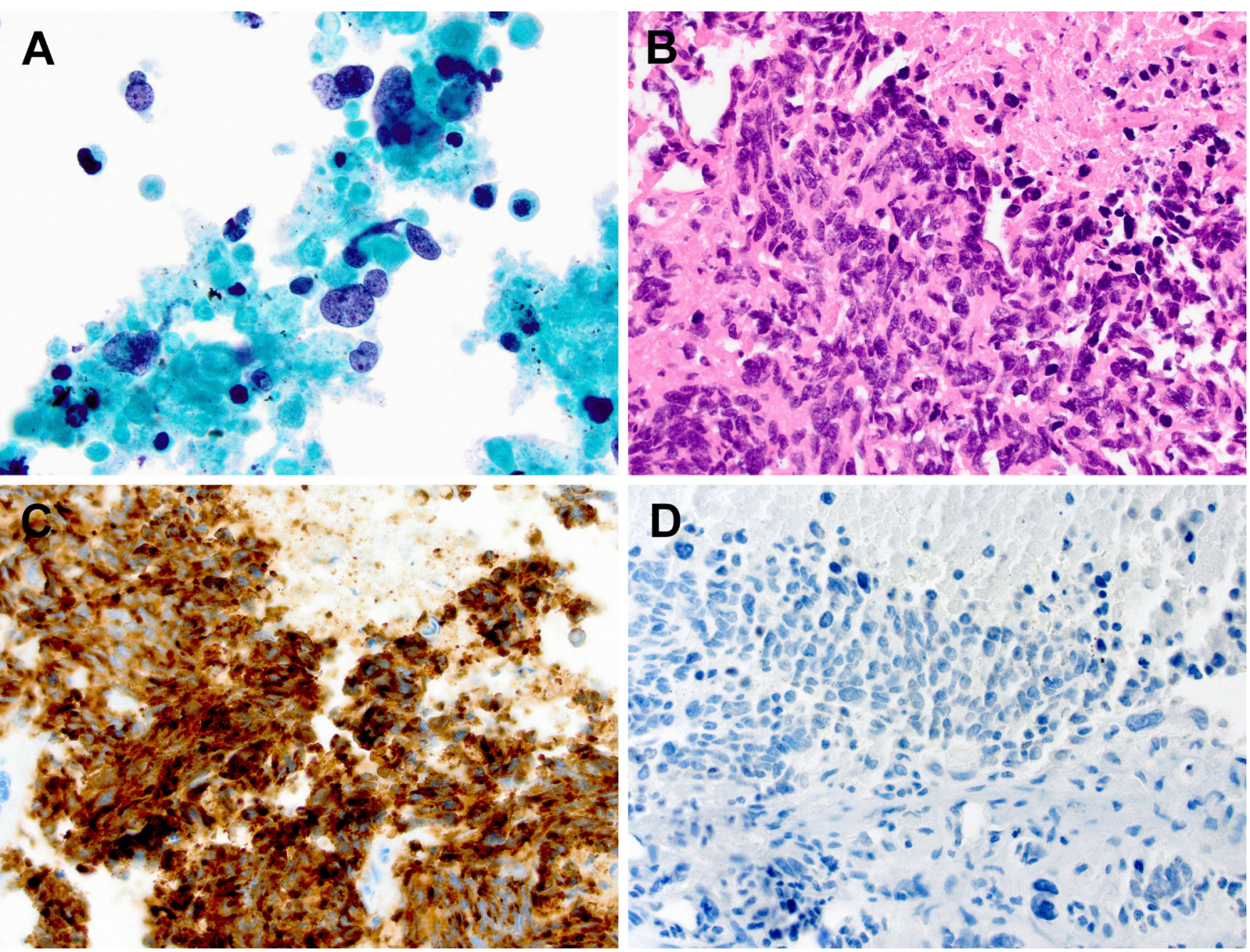

Figure 1.

Small cell carcinoma. A: Transbronchial needle aspiration of the right upper lobe lung mass demonstrates poorly differentiated tumor cells with scant cytoplasm, nuclear molding, and finely granular neuroendocrine-type chromatin, present in a background of tumor necrosis (ThinPrep, Papanicolaou stain, 1000x original magnification). B: Transbronchial biopsy of the right upper lobe mass highlights the prominent nuclear molding of the poorly differentiated tumor cells with frequent mitotic figures and tumor necrosis (Hematoxylin and Eosin stain, 600x objective original magnification). C: Immunohistochemical staining demonstrates strong cytoplasmic expression of the neuroendocrine marker synaptophysin (600x original magnification). The tumor was also positive for TTF-1 and chromogranin, negative for $\mathrm{p} 63$, and a Mib-1/Ki-67 proliferation marker was positive in $>90 \%$ of tumor cells (not shown), all consistent with small cell carcinoma. D: Immunohistochemical staining for EGFR demonstrates essentially no detectible cytoplasmic expression of EGFR (600x original magnification). 


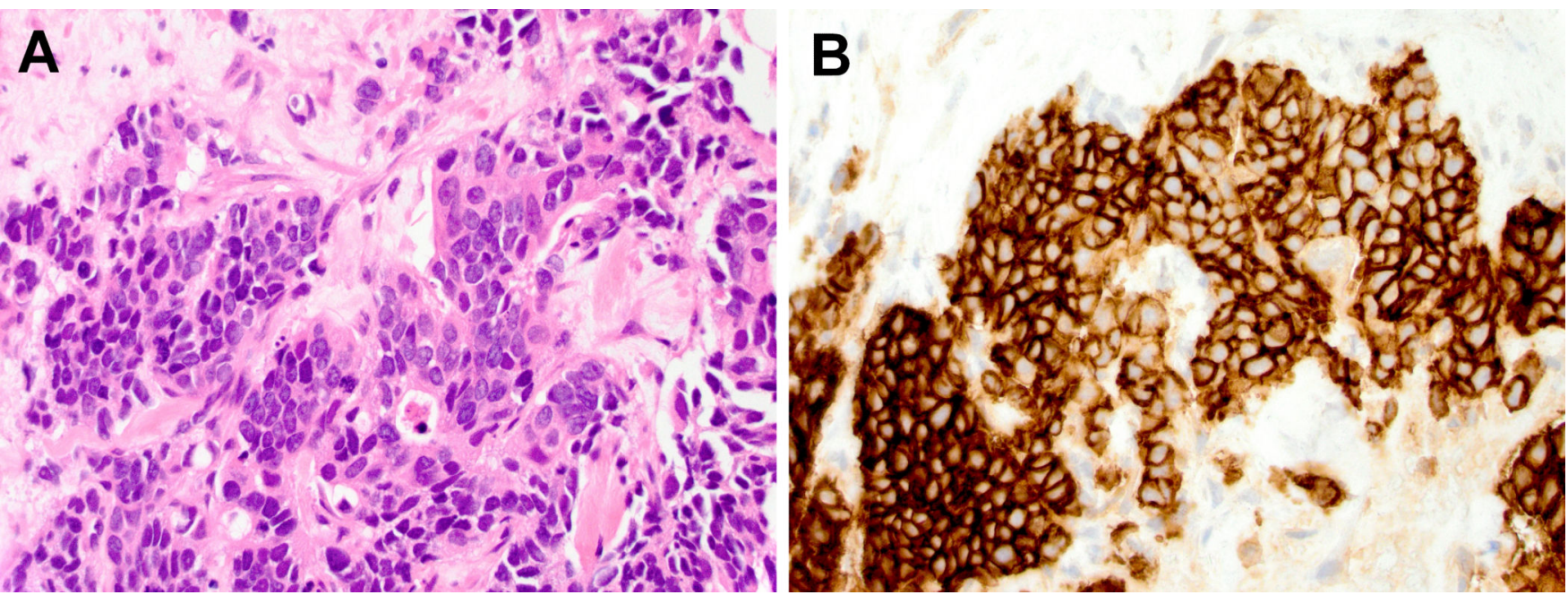

Figure 2.

Large cell neuroendocrine carcinoma. A: Ultrasound guided percutaneous core needle biopsy of the abdominal nodule demonstrates poorly differentiated tumor cells growing in solid nests and cords with moderate amounts of eosinophilic cytoplasm, round to oval nuclei with prominent nucleoli, some nuclear molding, and scattered single cell necrosis (Hematoxylin and eosin stain, 60x objective original magnification); no areas of glandular or squamous differentiation were identified. B: Immunohistochemical staining demonstrates strong diffuse cytoplasmic positivity for the neuroendocrine marker CD56 (600x original magnification). The tumor cells were also diffusely positive for synaptophysin, TTF-1, and cytokeratin 7; negative for chromogranin, cytokeratin 20; and a Mib-1/Ki-67 proliferation marker was positive in $>50 \%$ of tumor cells (not shown). In this small biopsy specimen, the findings are consistent with metastatic pulmonary large cell neuroendocrine carcinoma. 